\title{
Promotionspreis 2013 - Ausschreibung
}

Die Deutsche Gesellschaft für Ultraschall in der Medizin vergibt Förderpreise für hervorragende Dissertationen auf dem Gebiet des medizinischen Ultraschalls. Der Promotionspreis 2013 ist mit $€ 1000$,- dotiert und kann zweimal vergeben werden.

Für den DEGUM-Promotionspreis 2013 können Dissertationen berücksichtigt werden, die nach dem 31.12.2010 von einer deutschen Medizinischen Fakultät angenommen wurden. Habilitationsschriften sind von der Bewerbung ausgeschlossen.
Der Wissenschaftsbeirat der DEGUM bewertet die Arbeit auf der Grundlage von 2 Gutachten und entscheidet über die Preisvergabe, seine Entscheidung ist endgültig und nicht anfechtbar.

Die Bewerbung ist in elektronischer Form bis zum 19.04.2013 an die DEGUM-Geschäftsstelle zu richten. Der Bewerbung ist - ebenfalls in elektronischer Form - die Dissertation beizufügen.

München im November 2012

Dr. S. Nöldeke, Präsident der DEGUM

Prof. Dr. M. Görtler, Sekretär der DEGUM 